\title{
Componentes del amor y la satisfacción marital en casados y convivientes de Arequipa
}

Components of love and marital satisfaction in married and cohabitant people from Arequipa

\author{
Ana Lucía Núñez Cohello \\ Universidad Católica San Pablo, Arequipa, Perú
}

Recibido: 19-07-18

Aceptado: 03-10-18

\section{Resumen}

Esta investigación pretende medir y establecer la relación entre los componentes del amor de la teoría triangular de Sternberg y la satisfacción marital en parejas casadas y convivientes de la ciudad de Arequipa, mediante la aplicación de la Escala de Satisfacción Marital (Pick \& Andrade, 1988) y la Escala Triangular del Amor (Sternberg, 1989). Asimismo, compara los componentes del amor y la satisfacción de ambos modelos de relación conyugal en función del sexo y el estado civil. La muestra estuvo conformada por 296 personas que tenían una relación de pareja; de las cuales $12.2 \%$ está casado legalmente, $49.7 \%$ vive en matrimonio civil y religioso, y $38.2 \%$ mantiene una relación de convivencia. Se encontró una correlación positiva entre la variable amor y la satisfacción conyugal, y se hallaron diferencias significativas en intimidad, compromiso y pasión a favor de los casados, pero no hubo diferencias en satisfacción marital entre casados y convivientes.

Palabras clave: Satisfacción marital, amor, intimidad, pasión, compromiso.

\begin{abstract}
The present research aims to measure and establish the relation between the components of love in the triangular theory of Sternberg and marriage satisfaction in married couples and cohabitants of Arequipa city. The Marital Satisfaction Scale (Pick \& Andrade, 1988) and the Triangular Love Scale (Sternberg, 1989). In ad-
\end{abstract}


dition, our research compared the components of love and satisfaction of both marital relationship models according to sex and marital status. The sample consisted of 296 people who had a relationship, $12.2 \%$ get married only by law, $49.7 \%$ married legally and religiously, and $38.2 \%$ living together. A positive correlation was found between the variable love and marital satisfaction. Moreover, significant differences in intimacy, commitment and passion in favor of the married were found. However, there were no differences in marital satisfaction between married and cohabitants.

Keywords: Marital satisfaction, love, intimacy, passion, commitment.

\section{Introducción}

El ser humano, a lo largo de su vida, configura un gran número de relaciones interpersonales, entre las cuales se encuentra la relación de pareja (Blandón-Hincapié \& López-Serna, 2016). Esta es uno de los lazos afectivos más relevantes en la vida de todo hombre, puesto que, además de vincularnos en profundidad, permite satisfacer necesidades psicológicas de pertenencia y seguridad, así como también necesidades de orden íntimo y sexual (Mora, Gómez \& Rivera, 2013), lo que otorga un grado de intimidad psicológica que ninguna otra relación interpersonal puede ofrecer (Huston \& Levinger, 1978). El cuestionarse por las relaciones de pareja ha sido una constante a lo largo del tiempo, su estudio es de carácter nuclear y estructurante para la sociedad (Tobón, Vega \& Cuervo, 2012). Esto es porque las relaciones conyugales son un elemento fundamental en la conservación de la especie humana y favorecen el desarrollo del hombre desde el momento de su nacimiento (Félix \& Rodríguez, 2001), a través del cuidado, la formación de vínculos de apego, el amor y la educación en hábitos y valores.

Cuando dos personas deciden formalizar su relación, se crea una nueva unidad social conocida como sistema marital (Elsner, Montero, Reyes \& Zegers, 200o). Sin embargo, las transformaciones socioculturales, demográficas y económicas que se han experimentado a lo largo de los últimos años han afectado la estructura y la organización de la vida marital y familiar, de manera que si hace un par de años podía afirmarse que el matrimonio era la forma de organización conyugal predominante (Rodríguez, 2001), hoy en día es necesario poner en tela de juicio esta proposición, ya que la cohabitación como forma de unión y vida conyugal es ahora una realidad que forma parte de la identidad de las relaciones maritales de Latinoamérica (Lesthaeghe, 2014), donde Colombia, Panamá, Perú, Uruguay, Nicaragua y El Salvador son los países que reportan un mayor número de parejas de hecho (Flores, 2016). En lo que respecta a nuestro país, la Superintendencia Nacional de Registros Públicos (2017) afirmó que el número de cohabitantes en el Perú, hasta el 2017, ha- 
bía aumentado un $58 \%$ en relación con el año anterior, pasando de 853 parejas inscritas a 1348. Son muchas las personas que afirman que la convivencia debería favorecer el establecimiento de un matrimonio futuro, puesto que las parejas tendrían la oportunidad de conocer mejor a su cónyuge al tener más información de él, frente a aquellos que no han convivido (Boyle, 2006). Sin embargo, hasta el momento no hay evidencias empíricas suficientes que afirmen que las parejas que han vivido juntas antes de contraer matrimonio tengan una mejor relación, por el contrario, estudios demuestran que la mayoría dijo tener una relación descontenta; con menor compromiso, visión más individualista y mayor posibilidad de divorcio que aquellas parejas que deciden casarse en primera instancia (Rice, 200o). Está comprobado que el matrimonio es la forma de unión conyugal que otorga mayor cantidad de beneficios, puesto que concede un nivel de estabilidad en la pareja y los hijos que ningún otro modelo de vida marital puede otorgar (Huarcaya, 2011).

Junto con el aumento de las parejas de hecho, otro de los cambios más preocupantes es el incremento de las tasas de divorcio, como producto del mayor número de parejas que se sienten insatisfechas con su relación (Witherspoon Institute, 2008). Este es un inconveniente social relevante: "1 de cada 4 parejas reporta infelicidad en su matrimonio" (Lastra \& Umbo, 2017, p. 54).

La satisfacción marital puede ser definida como la percepción subjetiva que tiene cada miembro de una pareja de su relación y su cónyuge (Díaz-Loving, 1990) o como la apreciación global que cada uno elabora sobre el desarrollo de la relación (Villarreal, 2016). Ha sido y es un tema de interés tanto para terapeutas como para investigadores y profesionales dedicados al área de la salud, ya que aquellas parejas que reportan sentirse insatisfechas con su relación suelen manifestar consecuencias graves para su salud física y mental (Gottman \& Leverson, 1999), aumentando en un $35 \%$ las posibilidades de caer enfermo y, además, acortar la vida en un periodo medio de cuatro años (Gottman \& Silver, 2012). Por el contrario, se observa que aquellas parejas que presentan niveles adecuados de satisfacción marital reportan porcentajes de felicidad más altos, debido a las manifestaciones de afecto y apoyo que reciben (Villarreal, 2016). Incluso está comprobado que estas parejas se encuentran más satisfechas y felices en otros aspectos de su vida (laboral, social, familiar, etc.), puesto que tienen emociones y sentimientos más positivos (De Andrade, Rech \& Carnielli, 2015). Sin embargo, a pesar de conocer la relevancia del estudio de la satisfacción marital, en nuestro país, las investigaciones respecto a esta materia son muy escasas y recientes.

Asimismo, el estudiar las relaciones maritales implica abordar el tema del amor conyugal. Si bien es cierto que el amor entendido como aquel elemento que mantiene a dos personas unidas no se ha transformado ni se transformará con el paso 
de los años, la manera en que este se manifiesta y se incorpora en la sociedad sí ha cambiado (Fernández, 1998). Lamentablemente, el mundo en el que actualmente vivimos está envuelto en una cultura liberal y utilitarista, de modo que el amor se comprende a través de conceptos como "altruismo, filantropía o benevolencia" (García, 2016, p. 8). Ya no es considerado como sinónimo de donación total, sino como un mero sentimiento de cariño, atracción, semejanza o afinidad, adoptándose de esta manera un comportamiento posesivo o egoísta, en el que lo más importante es el yo, es sentirse bien con uno mismo, a pesar de que esto signifique sufrimiento para la otra persona.

De esta manera, el amor, que parecía ser el elemento que permitía a las parejas permanecer unidas ante cualquier dificultad, de repente se desvanece y lleva a la pareja a la separación y, por consiguiente, a la destrucción del sistema familiar (Nympha \& Gunda, 2015). Así, la relación conyugal percibida inicialmente como principio de bienestar físico y psicológico es ahora generadora de dolor y sufrimiento al verse deteriorada (Bastida, Valdez, Valor, González \& Rivera, 2017). Es por este motivo que estudiar el amor y conceptualizarlo correctamente, entendiéndolo como la renuncia a uno mismo y el sacrificio por el amado (Córdova, 2011), resulta de gran importancia, ya que a través de su investigación se podrá discernir lo que es correcto y anhelado en las relaciones humanas (Mônego \& Teodoro, 2011).

Entre la variedad de teorías expuestas acerca del amor romántico, la de Sternberg es una de las más prestigiosas, puesto que ha otorgado un amplio marco conceptual para la investigación científica del amor, incluso ha sido calificada como un adelanto representativo en el estudio empírico de este (Tzeng \& Gomez, 1992). A pesar de haber sido elaborada a fines de los años ochenta, esta teoría es hoy en día válida y confiable para el estudio de las relaciones conyugales (Villamizar, 2009). Así pues, como un intento de comprender la naturaleza del amor, Sternberg propuso la teoría triangular del amor, en la que este es entendido como un todo conformado por elementos muy complejos: intimidad, pasión y compromiso (García, Fuentes \& Sánchez, 2016).

\section{Intimidad}

La intimidad es conceptualizada como el sentimiento de permanecer juntos y manifestarse aprecio y cariño, sin que haya pasión ni compromiso de por medio (Almeida, 2013), de modo que se relaciona con términos como comodidad, felicidad y comunicación, lo que permite que los miembros de la relación tengan un vínculo sólido de proximidad y unión (Cassepp-Borges \& Pasquali, 2012). Sternberg y Grajek (1984) sostienen que la intimidad abarca sentimientos como deseo de incrementar el bienestar del cónyuge, satisfacción emocional al compartir con la persona 
amada, agradecimiento, confianza, comprensión, solidaridad, bienestar, soporte emocional, comunicación íntima y valoración del otro. Sin embargo, es necesario tener en cuenta que para que haya intimidad no es necesaria la presencia de todos los sentimientos aquí mencionados, ya que estos difieren de una persona a otra.

\section{Pasión}

Sternberg (1989) señala que los elementos que están incluidos en este componente son la atracción física, el apetito sexual y el romance. Además, es la pasión la que suscita agentes de motivación que dan lugar a la excitación o las necesidades como la autoestima, la potestad de uno mismo, la docilidad y la adhesión. Ficher (citado por Mazadiego \& Norberto, 2011), sostiene que este componente hace referencia al frenesí, amor abundante y desesperación que todos en algún momento han experimentado; aunque según Almeida (2013), la pasión hace referencia al deseo intenso de unión con la pareja y, en menor parte, a la sexualidad, la cual no necesariamente es de carácter carnal.

\section{Compromiso}

El compromiso hace alusión a dos elementos: la determinación de amar al otro y la de conservar dicho amor a través de los años (Sternberg, 1989); asimismo, en el compromiso existe la posibilidad de mantener la relación incluso sin que estén presentes la pasión y la intimidad (Haack \& Falcke, 2014). Muestra un curso único dentro de cada pareja, de manera que si una relación se desarrolla de forma satisfactoria, el compromiso irá en aumento, y esto permitirá el sostenimiento y la duración de la relación a través de los años. Si por el contrario la relación se deteriora, el compromiso podría incluso llegar a desvanecerse y acabar con la relación (Villamizar, 2009).

Está demostrado que tanto el amor como el tipo de unión marital influyen significativamente en la relación. De esta manera, Álvarez y García (2017) afirman que el amor favorece la satisfacción marital, de modo que en el 2015 Nympha y Guda llegaron a la conclusión de que cuando los niveles de intimidad, pasión y compromiso aumentan o disminuyen, la satisfacción conyugal también lo hace. Por otro lado, Tai, Baxter y Hewitt (2014) sostienen que la satisfacción conyugal también puede variar de acuerdo con el tipo de unión conyugal que se tenga: se encuentra que aquellos que mantienen una relación de convivencia presentan relaciones más pobres, son menos felices y perciben niveles más bajos de justicia y confianza, así como más conflictos de violencia; se muestra también que tanto los hombres como las mujeres que tuvieron una convivencia prematrimonial eran menos felices que aquellos que contrajeron matrimonio directamente (Brown, Manning \& Payne, 2014). 
Sternberg (1998) sugiere que las mujeres, al iniciar una relación afectiva, tienden a dirigir su atención al desarrollo de la intimidad y el compromiso, y no apuntan a una relación cargada de pasión. Así pues, cuando una mujer se enamora, tiene el deseo de estar junto a su pareja siempre, y desarrolla fuertes vínculos de intimidad, cercanía, confianza, apoyo y afectividad; de modo que una vez establecida una relación emocional sólida, recién podrá implicarse sexualmente con confianza y libertad, entonces, es necesario que antes se sienta querida, valorada y entendida por su pareja (Barrio \& Pinto, 2008). Por otro lado, los varones, al inicio de sus relaciones, suelen experimentar mayor interés por el desarrollo de la pasión, de modo que buscan involucrarse rápidamente en situaciones pasionales, motivo por el que en numerosos casos no necesitan desarrollar un lazo afectivo fuerte con su pareja (Sternberg, 1998). Barrios y Pinto (2008) sugieren que el varón no apunta inicialmente al compromiso y la intimidad porque tiene miedo de perder su independencia y su libertad, además que le atemoriza demostrar sus sentimientos y ser por ello ridiculizado.

Respecto a la satisfacción marital, Bounchard (2013) plantea que no hay diferencias significativas entre el grado de satisfacción marital experimentada por hombres y mujeres, dado que ambos se ven influidos de forma similar por los diversos acontecimientos que ocurren a lo largo de su vida marital. Sin embargo, Lavner y Bradbury (2010) corroboraron que las mujeres se preocupan más por tener un matrimonio satisfactorio que los hombres, esto porque las tareas que desempeñan y el número de obligaciones del hogar no son los mismos y es la mujer la que muchas veces se hace cargo del trabajo doméstico o el cuidado de los hijos, experimentando menos satisfacción marital. Por otro lado, Vera, Félix y Rodríguez (2001) sostienen que los hombres evidencian puntajes más altos de satisfacción que las mujeres.

El presente estudio pretende determinar la relación entre los niveles de satisfacción y amor registrados en parejas casadas (por matrimonio civil o religioso) y en convivientes de la ciudad de Arequipa, para posteriormente hacer una comparación en función del tipo de unión conyugal (los casados por la ley, los casados por la Iglesia y la ley, y los convivientes) para vislumbrar si efectivamente la convivencia otorga menos beneficios y niveles de satisfacción que el matrimonio civil o religioso.

\section{Metodología}

\section{Tipo y diseño de investigación}

Para la presente investigación se trabajó con un diseño transeccional correlacional (Hernández, Fernández \& Baptista, 2010) debido a que los datos de las variables de estudio fueron tomados en un solo momento, con el objetivo de determinar el 
grado de relación entre ambas. Asimismo, es un estudio de alcance correlacional, ya que busca medir las relaciones entre variables, y es también un estudio comparativo porque investiga la desigualdad entre dos grupos de personas (Ato, López \& Benavente, 2013).

\section{Muestra}

La muestra estuvo conformada por 296 personas que tenían una relación de pareja. El $43.9 \%$ de ellas eran varones y el 56.1 \%, mujeres; con una edad media de 40.71 años, y una desviación estándar de \pm 10.51 , dentro de un rango de 19 a 68 años de edad. Respecto al estado civil, el $12.2 \%$ era casado por civil, el $49.7 \%$ estaba casado por civil y por la Iglesia, mientras que el $38.2 \%$ mantenía una relación de convivencia. En promedio, las parejas tienen 13.24 años de relación de pareja, con una desviación estándar de \pm 9.65 , dentro de un rango que abarca desde menos de un año de pareja hasta 42 años de compromiso matrimonial. La muestra fue seleccionada de manera no probabilística, de diversas instituciones y a través de diversos medios. De este modo, el $13.2 \%$ de las personas en la muestra provienen de un movimiento religioso denominado Avanzada Católica, el $10.8 \%$ proviene de una empresa de transportes, el $20.9 \%$ son padres de familia de una institución educativa privada de Arequipa y el $18.2 \%$ proviene de una empresa manufacturera, todos ellos fueron evaluados en persona; el $36.8 \%$ restante fue seleccionado aleatoriamente y la evaluación fue a través de Internet.

\section{Instrumentos}

Escala de Satisfacción Marital (ESM)

La escala está compuesta por 24 ítems distribuidos en tres secciones diferentes: satisfacción con la interacción conyugal (1-10), satisfacción con los aspectos emocionales del cónyuge (11-15) y satisfacción con los aspectos organizacionales y estructurales de la relación (16-24) (Acevedo, Restrepo \& Tovar, 2007). Cada ítem de la escala posee tres opciones de respuesta: $1=$ Me gustaría muy diferente, $2=\mathrm{Me}$ gustaría algo diferente y 3 = Me gusta cómo está pasando (Pick \& Andrade, 1988). La consistencia para cada factor se obtuvo mediante un alfa de Cronbach, obteniendo los siguientes datos: Factor $1=0.81$, Factor $2=0.90$, Factor $3=0.85$ (Eguiluz, Calvo \& De la Orta, 2012), lo que indica que la escala es confiable. En relación con la validez, Gonzales (2011) obtuvo índices superiores a .677, lo que indica que la prueba posee una validez adecuada. 
Escala Triangular del Amor (ETA)

La escala es de tipo Likert, con un rango de 1 a 9 y consta de 45 ítems, 15 para cada uno de los componentes (intimidad, compromiso y pasión). La validez y la confiabilidad de la ETA han sido valoradas en la ciudad de Lima a través de diversos estudios. En primer lugar, la investigación realizada por Ventura y Caycho (2016) con estudiantes universitarios encontró que la escala total posee una confiabilidad de .o64; mientras que la dimensión de intimidad, .947; la de compromiso, .922; y la de pasión, .848 (Cicchetti, 1994). En cuanto a la validez, Ventura y Caycho (2016) reportan una correlación interfactorial positiva y moderada entre todas sus dimensiones. En un segundo estudio realizado a partir de una muestra de 20 personas, se obtuvo una consistencia interna de .986 (Bohórquez, 2017).

\section{Procedimiento}

Se aplicaron las encuestas y el consentimiento informado en cada una de las instituciones elegidas, donde se convocó a los participantes en salas de reuniones, otorgándoles alrededor de 20 minutos para completar las evaluaciones. Se leyeron las instrucciones en voz alta y todas las dudas fueron resueltas. Asimismo, se creó una versión virtual de la prueba, la cual fue compartida vía correo electrónico con diferentes conocidos que, se sabía, cumplían con los criterios de inclusión (ser mayor de edad y mantener una relación estable de pareja, sea casado o conviviente). La resolución de las escalas virtuales fue de manera anónima y personal, y las dudas fueron resueltas por la persona encargada a través del mismo medio utilizado para la evaluación.

\section{Resultados}

\section{Análisis descriptivo}

En primer lugar, tenemos la distribución de porcentajes de los niveles de satisfacción marital y sus respectivas dimensiones. En la Figura 1 se aprecia que la satisfacción respecto a la interacción con el cónyuge es alta para el $29.4 \%$ de la muestra, moderada para el $48 \%$ y baja para el $22.6 \%$. La satisfacción en cuanto a los aspectos emocionales del cónyuge es alta para el $26.4 \%$ de la muestra, moderada para el $46.6 \%$ y baja para el $27 \%$. La satisfacción respecto a los aspectos estructurales de la relación es alta para el $27.4 \%$ de la muestra, moderada para el $46.3 \%$ y baja para el $26.4 \%$; mientras que la satisfacción marital es alta para el $26.7 \%$ de la muestra, moderada para el $47.3 \%$ y baja para el $26 \%$. Ello supone que más de la tercera parte de la muestra se siente satisfecha con su relación de pareja. 


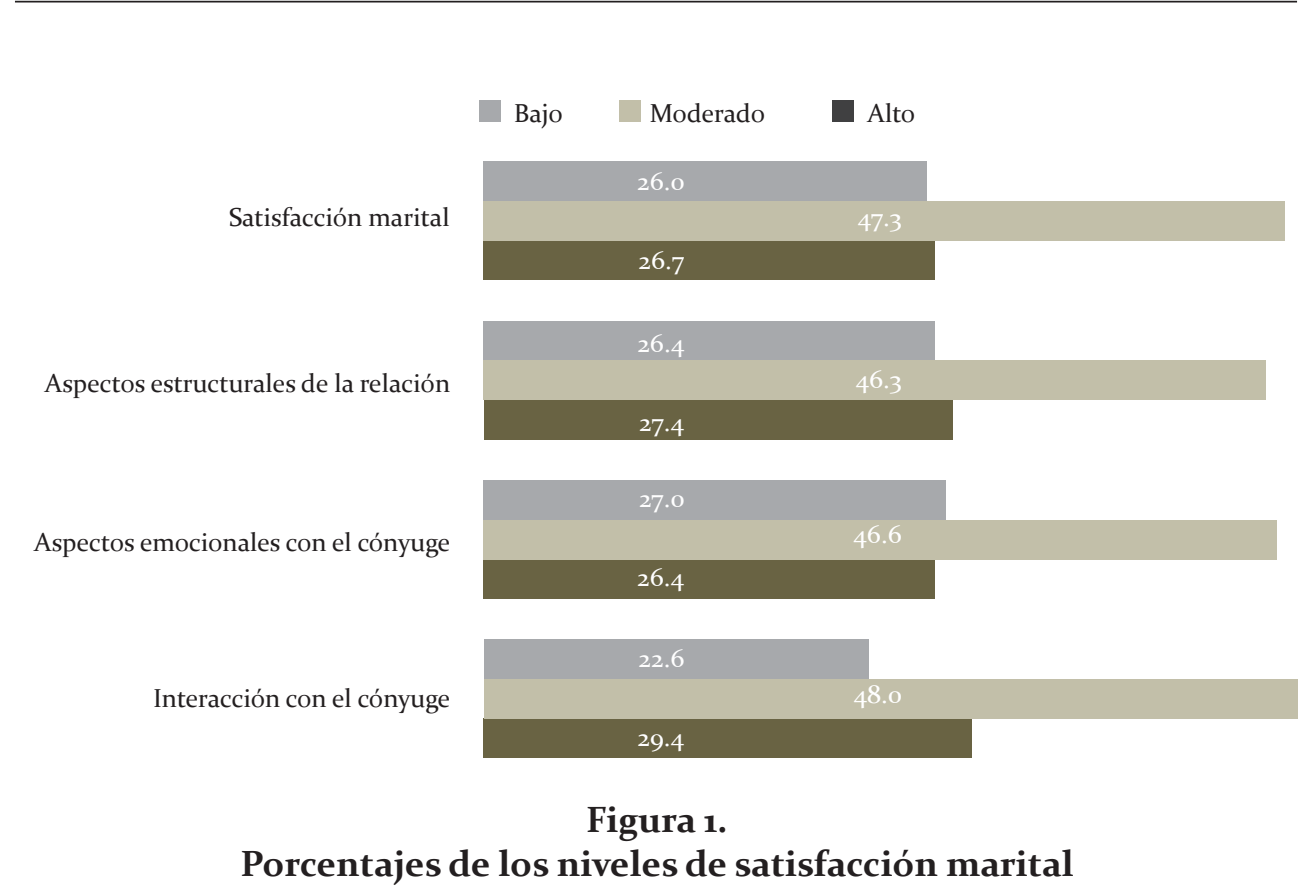

Con respecto a la variable amor, según la teoría de Sternberg, se tiene que el $28.4 \%$ tiene niveles bajos de intimidad; el $44.6 \%$, niveles moderados; y el $27 \%$, niveles altos. En la dimensión de compromiso, el $28.7 \%$ alcanza puntuaciones bajas; el $42.9 \%$, moderadas; y el $28.4 \%$, altas. En la dimensión de pasión, el $33.4 \%$ tiene un nivel bajo; el $40.5 \%$, moderado; y el $26 \%$, alto. Finalmente, con respecto a la puntuación total de amor, el $29.4 \%$ se ubica en un nivel bajo; el $45.6 \%$, en un nivel moderado; y el $25 \%$, en un nivel alto. Aquí también se observa que más de dos tercios de la muestra tienen niveles moderados y altos de intimidad, compromiso, pasión y amor.

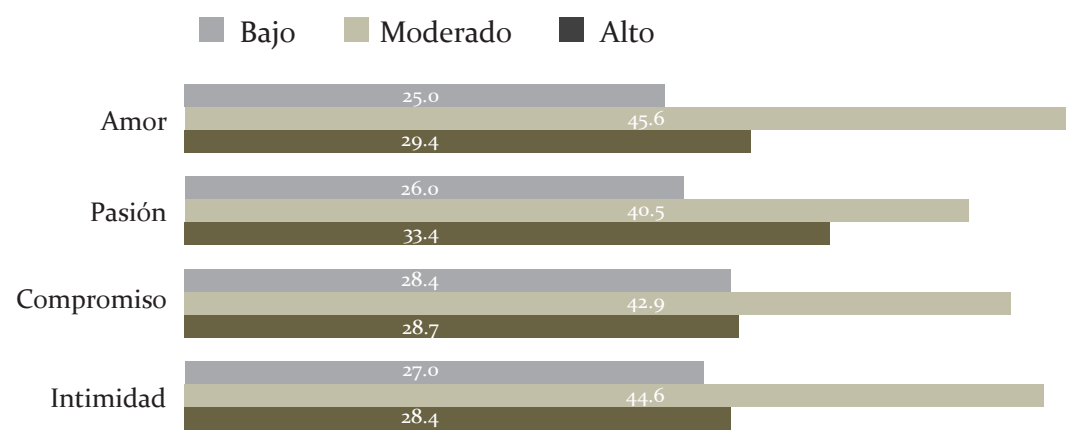

Figura 2

Porcentajes de los niveles de amor 


\section{Análisis comparativo}

En la Tabla 1, se tienen las comparaciones de las medias según el sexo, mediante la prueba t de Student. En ella se aprecia que en satisfacción con la interacción conyugal, en satisfacción con los aspectos organizacionales y estructurales de la relación, y en satisfacción marital, las mujeres presentan valores más altos que los varones. No obstante, en la variable amor y cada una de sus dimensiones, los varones presentan valores más altos que las mujeres; en todos estos casos, se hallaron diferencias significativas $(\mathrm{p}<.05)$.

Tabla 1.

\section{Comparaciones según sexo mediante la t de Student}

\begin{tabular}{lccccccc}
\hline & Sexo & $\mathrm{N}$ & Media & $\mathrm{DE}$ & $\mathrm{t}$ & $\mathrm{gl}$ & $\mathrm{p}$ \\
\hline Interacción & Varón & 130 & 20.530 & 6.235 & -2.986 & 289.792 & $\mathbf{0 . 0 0 3}$ \\
conyugal & Mujer & 166 & 22.843 & 7.066 & & & \\
Emociones del cón- & Varón & 130 & 8.269 & 2.188 & -1.418 & 288.175 & 0.157 \\
yuge & Mujer & 166 & 8.650 & 2.426 & & & \\
Aspectos & Varón & 130 & 14.076 & 4.117 & -2.112 & 289.745 & $\mathbf{0 . 0 3 6}$ \\
organizacionales & Mujer & 166 & 15.156 & 4.663 & & & \\
Satisfacción & Varón & 130 & 42.876 & 11.088 & -2.723 & 290.608 & $\mathbf{0 . 0 0 7}$ \\
marital & Mujer & 166 & 46.650 & 12.722 & & & \\
\multirow{2}{*}{ Intimidad } & Varón & 130 & 106.661 & 29.434 & 2.674 & 290.724 & $\mathbf{0 . 0 0 8}$ \\
& Mujer & 166 & 96.813 & 33.834 & & & \\
Compromiso & Varón & 130 & 82.076 & 23.778 & 2.613 & 288.316 & $\mathbf{0 . 0 0 9}$ \\
& Mujer & 166 & 74.433 & 26.419 & & & \\
Pasión & Varón & 130 & 38.453 & 11.835 & 2.869 & 289.443 & $\mathbf{0 . 0 0 4}$ \\
& Mujer & 166 & 34.247 & 13.346 & & & \\
Amor & Varón & 130 & 227.192 & 62.951 & 2.779 & 289.576 & $\mathbf{0 . 0 0 6}$ \\
& Mujer & 166 & 205.494 & 71.119 & & & \\
\hline
\end{tabular}

En la Tabla 2, se observa el análisis de varianza de las variables de estudio según el estado civil de los participantes. Se aprecia que en la satisfacción marital y sus respectivas dimensiones no se registraron diferencias significativas, pero en la variable amor y las dimensiones de intimidad, compromiso y pasión se obtuvieron valores significativos a favor de los casados por la Iglesia, mientras que los convivientes obtuvieron valores superiores en comparación con las parejas casadas solo por la ley. 
Tabla 2.

Comparaciones según el estado civil mediante el análisis de varianza

\begin{tabular}{|c|c|c|c|c|c|c|}
\hline & Estado civil & Medias & $\mathrm{gl}$ & $\begin{array}{c}\text { Media } \\
\text { cuadrática }\end{array}$ & $\mathrm{F}$ & Sig. \\
\hline \multirow{3}{*}{$\begin{array}{l}\text { Interacción } \\
\text { conyugal }\end{array}$} & Casado por la ley & 20.388 & 2 & 91.214 & 1.985 & 0.139 \\
\hline & Casado por la Iglesia & 21.489 & 293 & $45 \cdot 951$ & & \\
\hline & Conviviente & 22.725 & 295 & & & \\
\hline \multirow{3}{*}{$\begin{array}{l}\text { Emociones } \\
\text { del cónyuge }\end{array}$} & Casado por la ley & 8.027 & 2 & 4.664 & 0.859 & 0.425 \\
\hline & Casado por la Iglesia & 8.496 & 293 & 5.429 & & \\
\hline & Conviviente & 8.610 & 295 & & & \\
\hline \multirow{3}{*}{$\begin{array}{l}\text { Aspectos } \\
\text { organizacionales }\end{array}$} & Casado por la ley & 13.444 & 2 & 51.378 & 2.615 & 0.075 \\
\hline & Casado por la Iglesia & $14 \cdot 510$ & 293 & 19.650 & & \\
\hline & Conviviente & $15 \cdot 300$ & 295 & & & \\
\hline \multirow{3}{*}{$\begin{array}{l}\text { Satisfacción } \\
\text { marital }\end{array}$} & Casado por la ley & 41.861 & 2 & 347.404 & 2.372 & 0.095 \\
\hline & Casado por la Iglesia & 44.496 & 293 & 146.461 & & \\
\hline & Conviviente & 46.637 & 295 & & & \\
\hline \multirow{3}{*}{ Intimidad } & Casado por la ley & 90.861 & 2 & 4031.716 & $3 \cdot 942$ & 0.020 \\
\hline & Casado por la Iglesia & 105.884 & 293 & 1022.716 & & \\
\hline & Conviviente & 98.238 & 295 & & & \\
\hline \multirow{3}{*}{ Compromiso } & Casado por la ley & 69.444 & 2 & 3666.767 & 5.806 & 0.003 \\
\hline & Casado por la Iglesia & 82.585 & 293 & 631.514 & & \\
\hline & Conviviente & 74.212 & 295 & & & \\
\hline \multirow{3}{*}{ Pasión } & Casado por la ley & 31.250 & 2 & 914.954 & $5 \cdot 713$ & 0.004 \\
\hline & Casado por la Iglesia & 38.367 & 293 & 160.141 & & \\
\hline & Conviviente & 34.681 & 295 & & & \\
\hline \multirow{3}{*}{ Amor } & Casado por la ley & 191.555 & 2 & 23688.428 & 5.208 & 0.006 \\
\hline & Casado por la Iglesia & 226.836 & 293 & 4548.601 & & \\
\hline & Conviviente & 207.132 & 295 & & & \\
\hline
\end{tabular}

\section{Análisis correlacional}

Para la realización del análisis correlacional se aplicó la prueba de correlación de Pearson (ver Tabla 3). La satisfacción con la interacción conyugal se correlacionó de manera positiva y alta con la satisfacción con los aspectos organizacionales y estructurales de la relación y con la satisfacción marital, y de manera más moderada con la satisfacción con las emociones del cónyuge. Sin embargo, la satisfacción 
con la interacción conyugal se correlacionó de manera negativa y moderada con las dimensiones de intimidad, compromiso, pasión y amor. La satisfacción con las emociones del cónyuge se correlacionó de manera moderada alta y positiva con la satisfacción con los aspectos organizacionales de la relación y la satisfacción marital, pero de manera negativa y baja moderada con las dimensiones de la variable amor. La dimensión de satisfacción con los aspectos organizacionales de la relación se correlacionó alta y positivamente con la satisfacción marital, pero de manera baja y negativa con la variable amor y sus dimensiones. La satisfacción marital también se correlacionó de manera negativa y moderada con las dimensiones de la variable amor y su puntuación total. Finalmente, se tiene que la variable amor y sus respectivas dimensiones se correlacionan de manera alta, positiva y significativa entre sí. Se debe aclarar que los valores negativos reportados se producen porque la calificación de la Escala de Satisfacción Marital ofrece puntuaciones descendentes si se desea que se mantenga la relación como viene ocurriendo.

Tabla 3.

Correlaciones entre variables mediante la prueba de Pearson

\begin{tabular}{|c|c|c|c|c|c|c|c|c|}
\hline & 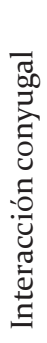 & 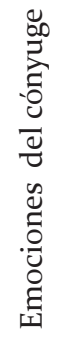 & 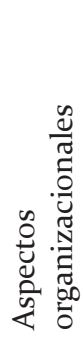 & 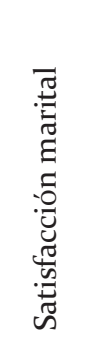 & 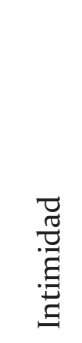 & 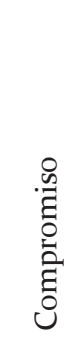 & 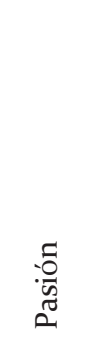 & 完 \\
\hline Interacción conyugal & 1 & .527 & .811 & .958 & -.303 & -.313 & -.246 & -.306 \\
\hline Emociones del cónyuge & & 1 & .501 & .670 & -.252 & -.226 & -.235 & -.247 \\
\hline Aspectos organizacionales & & & 1 & $.916^{* *}$ & -.262 & -.272 & -.194 & -.262 \\
\hline Satisfacción marital & & & & 1 & -.314 & -.318 & -.253 & -.315 \\
\hline Intimidad & & & & & 1 & .961 & .833 & .988 \\
\hline Compromiso & & & & & & 1 & .823 & .982 \\
\hline Pasión & & & & & & & 1 & .889 \\
\hline Amor & & & & & & & & 1 \\
\hline
\end{tabular}




\section{Análisis predictivo}

El análisis predictivo implicó, en primer lugar, hacer un análisis de regresión tomando los componentes del amor como variables predictoras de la satisfacción marital. Sin embargo, el modelo obtenido no fue significativo, ni se encontraron valores de importancia en los coeficientes beta estandarizados de las dimensiones del amor, como predictores de la satisfacción marital. Esto supone que, aunque ambas variables están relacionadas entre sí, el amor no predice la satisfacción marital. Por ello se realizaron modelos de regresión que plantearan predicciones en la dirección opuesta.

De este modo, como se observa en la Tabla 4, al efectuar el análisis de regresión, tomando como variables predictoras las dimensiones de satisfacción marital y la dimensión de intimidad de la variable amor como dependiente, se encontró que el modelo de regresión fue significativo y que la satisfacción, únicamente en su componente de interacción conyugal, predice la intimidad del amor en la relación de pareja de manera negativa y significativa $(\mathrm{p}=0.026)$.

\section{Tabla 4 . \\ Análisis de regresión Modelo 1}

\begin{tabular}{|c|c|c|c|c|c|}
\hline \multirow[t]{2}{*}{ Dimensión } & \multicolumn{2}{|c|}{$\begin{array}{l}\text { Coeficientes no } \\
\text { estandarizados }\end{array}$} & \multirow{2}{*}{$\begin{array}{c}\begin{array}{c}\text { Coeficientes } \\
\text { estandarizados }\end{array} \\
\text { Beta } \\
\end{array}$} & \multirow[t]{2}{*}{$\mathrm{t}$} & \multirow[t]{2}{*}{ Sig. } \\
\hline & B & Desv. error & & & \\
\hline (Constante) & 140.889 & $7 \cdot 442$ & & 18.931 & 0.000 \\
\hline Interacción conyugal & -1.040 & 0.463 & -0.219 & -2.244 & 0.026 \\
\hline Emociones del cónyuge & -1.738 & 0.914 & -0.125 & -1.902 & 0.058 \\
\hline $\begin{array}{l}\text { Aspectos } \\
\text { organizacionales }\end{array}$ & -0.157 & 0.695 & -0.022 & -0.226 & 0.821 \\
\hline
\end{tabular}

$\mathrm{p}=$ o.ooo; $\mathrm{VD}=$ Intimidad

En la Tabla 5, se observa el modelo de regresión, en el que las variables predictoras son las dimensiones de la satisfacción marital y el compromiso, la variable dependiente. Aquí se reporta que el modelo fue significativo y la satisfacción únicamente en su componente de interacción conyugal tuvo un impacto negativo y significativo sobre la dimensión de compromiso de la variable amor $(\mathrm{p}=0.013)$. 
Tabla 5 .

Análisis de regresión Modelo 2

\begin{tabular}{|c|c|c|c|c|c|}
\hline \multirow[t]{2}{*}{ Dimensión } & \multicolumn{2}{|c|}{$\begin{array}{l}\text { Coeficientes no } \\
\text { estandarizados }\end{array}$} & \multirow{2}{*}{$\begin{array}{c}\text { Coeficientes } \\
\text { estandarizados } \\
\text { Beta } \\
\end{array}$} & \multirow[t]{2}{*}{$\mathrm{t}$} & \multirow[t]{2}{*}{ Sig. } \\
\hline & B & Desv. error & & & \\
\hline (Constante) & 108.131 & 5.883 & & 18.381 & 0.000 \\
\hline Interacción conyugal & -0.913 & 0.366 & -0.243 & -2.492 & 0.013 \\
\hline Emociones del cónyuge & -0.891 & 0.723 & -0.081 & -1.233 & 0.219 \\
\hline $\begin{array}{l}\text { Aspectos organiza- } \\
\text { cionales }\end{array}$ & -0.195 & 0.549 & -0.034 & -0.355 & 0.723 \\
\hline
\end{tabular}

$\mathrm{p}=$ o.ooo $; \mathrm{VD}=$ Compromiso

Finalmente, se tiene que en el último modelo de regresión las variables predictoras son las dimensiones de la satisfacción marital y la variable dependiente es la pasión. Se observa que el modelo de regresión es significativo, y que es la dimensión satisfacción con las emociones del cónyuge la que tuvo un efecto negativo y significativo en la variable pasión $(\mathrm{p}=0.025)$.

Tabla 6.

\section{Análisis de regresión Modelo 3}

\begin{tabular}{|l|c|c|c|c|c|}
\hline \multirow{2}{*}{ Dimensión } & \multicolumn{2}{|c|}{$\begin{array}{c}\text { Coeficientes no } \\
\text { estandarizados }\end{array}$} & $\begin{array}{c}\text { Coeficientes } \\
\text { estandarizados }\end{array}$ & t & \multirow{2}{*}{ Sig. } \\
\cline { 2 - 5 } & \multicolumn{1}{|c|}{$\mathrm{B}$} & Desv. error & Beta & & \\
\hline (Constante) & 49.575 & 3.006 & -0.207 & -2.086 & 0.038 \\
Interacción conyugal & -0.390 & 0.187 & -0.151 & -2.254 & $\mathbf{0 . 0 2 5}$ \\
Emociones del cónyuge & -0.832 & 0.369 & 0.050 & 0.510 & 0.610 \\
Aspectos organizacionales & 0.143 & 0.281 & &
\end{tabular}

$\mathrm{p}=$ o.ooo $; \mathrm{VD}=$ Pasión

De estos análisis, se puede concluir que es la satisfacción marital la que tiene efecto sobre el amor; puntualmente, la satisfacción con la interacción conyugal tiene efecto sobre la intimidad y el compromiso, y la satisfacción con los aspectos emocionales del cónyuge tiene efecto sobre la pasión. 


\section{Discusión}

$\mathrm{Al}$ estudiar la satisfacción marital en parejas casadas y convivientes de la ciudad de Arequipa, se observó que un $\mathbf{4 7 . 2} \%$ de la muestra, es decir, casi la mitad de los encuestados, presenta un nivel moderado, mientras que solo un $26.6 \%$ reporta niveles altos de satisfacción marital, y el componente con índices más elevados es el relacionado con la interacción conyugal. Asimismo, son las mujeres quienes se sienten más satisfechas con su relación, lo que contradice la teoría postulada por Lavner y Bradbury (2010), que afirma que las mujeres tendrían índices bajos de satisfacción conyugal debido a que son ellas quienes tienen un mayor número de obligaciones domésticas, tales como la limpieza de la casa y el cuidado de los hijos. La afirmación de estos autores podría verse refutada también por la creciente inserción de la mujer en el mundo laboral, de forma que las tareas domésticas son repartidas con más equidad entre ambos miembros de la pareja, o son designadas a una tercera persona. Así, aquellos acontecimientos que podrían afectar la satisfacción femenina se habrían eliminado o reducido.

En cuanto a la puntuación total del amor, el $45.6 \%$ se encuentra en un nivel intermedio; mientras que un $25 \%$, en un nivel alto; y el $29.4 \%$, en un nivel bajo. Asimismo, el componente de compromiso es el que refleja niveles más elevados, seguido por la intimidad y finalmente la pasión. Los hombres son quienes presentan porcentajes más altos de intimidad, compromiso y pasión, al igual que lo presentado por Mazadiego y Norberto en el 2011. Este resultado es opuesto al de la investigación de Bohórquez (2017), realizada en Lima, en la que no se encontraron diferencias significativas en la variable amor según el sexo, de modo que podríamos concluir que los resultados aquí obtenidos serían características propias de los hombres arequipeños.

Por otro lado, al efectuar el análisis correlacional mediante la prueba de correlación de Pearson, se observó que todos los componentes del amor de la teoría de Sternberg se relacionan con la satisfacción marital y sus tres dimensiones, resultados que también fueron obtenidos en las investigaciones de Acker y Davis (1992), Lemieux y Hale (1999), Madey y Rodgers (2009), Overbeek, Ha, Scholte, De Kempy y Engewls (2007), y Sternberg (1989). De manera que se puede llegar a la conclusión de que a mayores niveles de intimidad, compromiso y pasión, mayor satisfacción marital. Así pues, es importante que las parejas no descuiden la intimidad, el compromiso y la pasión dentro de su relación, pues estas dimensiones afectarán de forma significativa su felicidad, acrecentándola o disminuyéndola.

Asimismo, la investigación no reportó diferencias significativas entre casados y convivientes en la variable de satisfacción marital, resultados que contradicen a varias de las investigaciones hasta ahora realizadas, en las que se afirma que las 
parejas que cohabitan se sentirían más descontentas con su relación (Bouchard, 2006; Myers, 2000; Teachman, 2003; Rhoades, Stanley \& Markman, 2012; Brown, Manning \& Payne, 2014). Entonces, se podría afirmar que hoy en día la cohabitación aporta los mismos efectos satisfactorios que el matrimonio, pues como postula Musick y Bumpass (2012), pareciera que las diferencias documentadas entre ambas formas de vida marital podrían estar atenuándose debido a la generalización de la cohabitación en varias partes de nuestro territorio, donde la convivencia ya no es vista como una prueba frente al matrimonio, sino más bien como un estilo de vida que abre las puertas a la relación conyugal.

Sin embargo, sí se encontraron diferencias significativas en la variable amor y sus tres dimensiones (intimidad, compromiso y pasión) a favor de los casados. Se concluye que las parejas de hecho podrían proveer menos apoyo a su cónyuge, a comparación de aquellos que se encuentran casados (Musick y Bumpass, 2012). Asimismo, dicho resultado concuerda con la conclusión a la que llegaron Pollard \& Harris (2013) en su investigación, en la que observaron que $41 \%$ de los hombres y $26 \%$ de las mujeres que mantienen una relación de convivencia no se encuentran ciento por ciento comprometidos con su relación, puesto que, como afirmaron Niehuis, Reifamn y Lee en el 2013, una vez que la pareja comienza a convivir el compromiso disminuye, dado que el objetivo principal que tiene una pareja al iniciar su convivencia no es el de una relación para toda la vida (Rodríguez-Vignoli, 2005); así, el tiempo de convivencia se convierte en una especie de ensayo. Del mismo modo, Lachmann (2006) encontró que las parejas casadas tienen puntajes más altos en los componentes de pasión e intimidad.

Finalmente, se llegó a la conclusión de que la satisfacción marital tiene un efecto predictivo en el amor, a diferencia de lo sugerido por Díaz-Guerrero (2003), quien afirma que la felicidad brota del amor. Puntualmente, las relaciones conyugales de Arequipa esperan sentirse satisfechas con el interés que su cónyuge pone en la relación para desarrollar intimidad y compromiso. Asimismo, es necesario que tengan experiencias afectivas y emocionales satisfactorias para dar lugar a la pasión; de modo que el inicio de la intimidad sexual en una pareja se vería profundamente ligado a la complacencia emocional con el otro. Este resultado también podría explicar el aumento de divorcios en nuestra ciudad, puesto que si un miembro de la pareja no se siente satisfecho, no habría amor en la relación y por lo tanto esta terminaría por disolverse. Igualmente, los resultados son el reflejo de la tendencia cultural actual en la que se nos infunde la idea de que lo más importante es sentirse bien con uno mismo, disfrutar siempre y de forma inmediata. De acuerdo con esta idea, cualquier dificultad o signo de aprieto es rechazado; de modo que cuando una pareja no se siente bien en todos los aspectos de su relación, se privará de dar su ciento por ciento para sacar adelante su amor y optará por la solución más fácil, que es la separación o el divorcio. 


\section{Referencias}

Acevedo, V., Restrepo, L., \& Tovar, J. (2007). Parejas satisfechas de larga duración en la ciudad de Cali. Pensamiento Psicológico, 3(8), 85-107.

Acker, M., \& Davis, M. (1992). Intimacy, passion, and commitment in Chinese and US American romantic relationships. International Journal of Intercultural Relations, 25(3), 329-342.

Almeida, A. (2013). Las ideas del amor de R. J. Sternberg: la teoría triangular y la teoría narrativa del amor. Familia, 46, 57-86.

Álvarez, E., \& García, M. (2017). Estilos de amor y culpa como predictores de la satisfacción marital en hombres y mujeres. Enseñanza e Investigación en Psicología, 22(1), 76-85.

Ato, M., López, J., \& Benavente, A. (2013). Un sistema de clasificación de los diseños de investigación en psicología. Anales de Psicología, 29(3), 1038-1059.

Bastida, R., Valdez, J., Valor, I., González, N., \& Rivera, S. (2017). Satisfacción marital y estado civil como factores protectores de la depresión y ansiedad. Revista Argentina de Clínica Psicológica, 26(1), 95-102.

Blandón-Hincapié, A., \& López-Serna, L. (2016). Comprensiones sobre pareja en la actualidad: jóvenes en busca de estabilidad. Revista Latinoamericana de Ciencias Sociales, Niñez y Juventud, 14(1), 505-517.

Bohórquez, B. (2017). Parejas virtuales y la teoría triangular del amor en jóvenes de Lima Metropolitana (tesis de pregrado). Pontificia Universidad Católica del Perú, Lima, Perú.

Bouchard, G. (2006). Cohabitation versus marriage: the role of dyadic adjustment in relationship dissolution. Journal of Divorce \& Remarriage, 46(1-2), 107-117.

Boyle, P. (2006). Does cohabitation prior to marriage raise the risk of marital dissolution and does this effect vary geographically? (MPIDR Working Paper WP 2006-051). Max Planck Institute for Demographic Research. Recuperado de http://www.demogr.mpg.de/papers/working/wp-2006-051.pdf

Brown, S., Manning, W., \& Payne, K. (2014). Relationship quality among cohabiting versus married couples. Ohio: National Center for Family and Marriage Research.

Cassepp-Borges, V., \& Pasquali, L. (2012). A redução de itens como uma alternativa para a Escala Triangular do Amor. Psicologia, 28(2), 11-20.

Cicchetti, D. (1994). Guidelines, criteria, and rules of thumb for evaluating normed and standardized assessment instruments in psychology. Psychological Assesments, 6, 284-290.

Córdova, N. (2011). El noviazgo: ¿pasatiempo o compromiso? Recuperado de https://parroquiaicm.wordpress.com/2011/11/27/el-noviazgo-pasatiempo-ocompromiso/. 
De Andrade, A., Rech, J., \& Carnielli, A. (2015). Relationship satisfaction in young adults: gender and love dimensions. Interpersona. An International Journal on Personal Relationships, 9(1), 19-31.

Díaz-Guerrero, R. (2003). Psicología del mexicano. Descubrimiento de la etnopsicología. México: Trillas.

Díaz-Loving, R. (1990). Configuración de los factores que integran la relación de pareja. Revista de Psicología Social en México, 3, 133-143.

Eguiluz, L., Calvo, R., \& De la Orta, D. (2012). Relación entre la percepción de la satisfacción marital, sexual y la comunicación en parejas. Revista Peruana de Psicología y Trabajo Social, 1, 15-28.

Elsner, P., Montero, M., Reyes, C., \& Zegers, B. (200o). La familia: una aventura. Santiago de Chile: Ediciones Universidad Católica de Chile.

Félix, M., \& Rodríguez, A. (2001). Relación existente entre el estrés en la crianza y el número de hijos asociado a la satisfacción marital (tesis de pregrado). Instituto Tecnológico de Sonora, Sonora, México.

Fernández, C. (1998). El arte de amar: un análisis sociológico. Reis, 84(98), 125-146.

Flores, E. (2016). Sistemas familiares y transiciones a la unión y al primer hijo en América Latina (tesis doctoral). Universidad Autónoma de Barcelona, Barcelona, España.

García, F., Fuentes, R., \& Sánchez, A. (2016). Amor, satisfacción en la pareja y resolución de conflictos en adultos jóvenes. Ajayu. Órgano de Difusión Científica del Departamento de Psicología de la Universidad Católica Boliviana "San Pablo", 14(2), 284-302.

Gonzales, H. (2011). Componentes de amor y satisfacción en padres de familia de la institución educativa Santa Ana (tesis de pregrado). Universidad César Vallejo, Trujillo, Perú.

Gottman, J., \& Levenson, R. (1999). Rebound from marital conflict and divorce prediction. Family Process, 38, 287-292.

Gottman, J., \& Silver, N. (2012). Siete reglas de oro para vivir en pareja. Barcelona: Random House Mondadori.

Haack, K., \& Falcke, D. (2014). Love and marital quality in romantic relationships mediated and non-mediated by internet. Paidéia, 24(57), 105-113.

Hernández, R., Fernández, C., \& Baptista, P. (2010). Metodología de la investigación (5. ${ }^{a}$ ed.). México: McGraw-Hill.

Huarcaya, G. (2011). La familia peruana en el contexto global. Impacto de la estructura familiar y la natalidad en la economía y el mercado. Mercurio Peruano: Revista de Humanidades, 524, 13-21.

Huston, L., \& Levinger, G. (1978). Interpersonal attraction and relationships. Annual Review of Psychology, 29, 115-157. 
Lachmann, I. (2006). Estilos de apego y componente del amor (tesis de pregrado). Universidad Rafael Urdaneta, Maracaibo, Venezuela.

Lastra, H., \& Umbo, Y. (2017). Estilos de apego emocional y satisfacción marital en los padres de familia del distrito de Huachón de Pasco. Revista de Investigación Apuntes Psicológicos, 2(1), 53-62.

Lavner, J., \& Bradbury, T. (2010). Patterns of change in marital satisfaction over the newlywed years, Journal of Marriage and Family, 75(5), 1171-1187.

Lemieux, R., \& Hale, J. (1999). Intimacy, passion, and commitment in young romantic relationships: Successfully measuring the Triangular Theory of Love. Psychological Reports, 85(2), 497-503.

Lesthaeghe, R. (2014). The second demographic transition: a concise overview of its development. Proceedings of the National Academy of Sciences, 111(51), 1811218115 .

Madey, S. F., \& Rodgers, L. (2009). The effect of attachment and Sternberg's Triangular Theory of Love on relationship satisfaction. Individual Differences Research, 7(2), 76-84.

Mazadiego, T., \& Garcés, J. (2011). El amor medido por la Escala Triangular de Sternberg. Psicolatina, 22, 1-10.

Mônego, B. G., \& Teodoro, M. L. M. (2011). A teoria triangular do amor de Sternberg e o modelo dos cinco grandes fatores. Psico-USF, 16(1), 97-105.

Mora, M., Gómez, M., \& Rivera, M. (2013). La satisfacción marital y los recursos psicológicos en las parejas con y sin hijos pequeños en pro del bienestar familiar. Uaricha, 10(22), 79-96.

Musick, K., \& Bumpass, L. (2012). Reexamining the case for marriage: Union formation and changes in well-being. Journal of Marriage and Family, 74, 1-18.

Myers, D. (2000). The funds, friends, and faith of happy people. American Psychologist, 55(1), 56-67.

Niehuis, S., Reifman, A., \& Lee, K. (2013). Disillusionment in cohabiting and married couples: a national study. Journal of Family Issues, 20(10), 1-13.

Nympha, M., \& Guda, I. (2015). Indicators of Marital Satisfaction of Batangueño Couples: Components of Love and the Other External Factors in Marriage. International Journal of Information and Education Technology, 5(1), 6o-67.

Overbeek, G., Ha, T., Scholte, R., De Kemp, R., \& Engels, R. (2006). Brief report: Intimacy, passion, and commitment in romantic relationships. Validation of a "triangular love scale" for adolescents. Journal of Adolescence, 30(3), 523-528.

Pick, S., \& Andrade, P. (1988). Desarrollo y validación de la Escala de Satisfacción Marital. Psiquiatría, 4(1), 9-20.

Pollard, M., \& Harris, K. (2013). Cohabitation and marriage intensity. Carolina del Norte: NIA funded RAND Center for the Study of Aging. 
Rhoades, G., Stanley, S., \& Markman, H. (2012). The impact of the transition to cohabitation on relationship functioning: Cross-sectional and longitudinal findings. Journal of Family Psychology, 26, 348-358.

Rice, P. (2000). Adolescencia, desarrollo, relaciones y cultura. Madrid: Prentice Hall. Rodríguez, T. (2001). Las razones del matrimonio. Representaciones, relatos de vida y sociedad. México: Universidad de Guadalajara.

Rodríguez-Vignoli, J. (2005). Unión y cohabitación en América Latina: ¿modernidad, exclusión, diversidad? Serie Población y Desarrollo, 57, 5-40.

Sternberg, R. (1989). El triángulo del amor. Barcelona: Paidós.

Sternberg, R., \& Grajek, S. (1984). The nature of love. Journal of Personality and Social Psychology, 47(2), 312-329.

Tai, T., Baxter, J., \& Hewitt, B. (2014). Do co-residence and intentions make a difference? Relationship satisfaction in married, cohabiting, and living apart together couples in four countries. Demographic Research, 31, 71-104.

Teachman, J. (2003). Premarital sex, premarital cohabitation, and the risk of subsequent marital dissolution among women. Journal of Marriage and Family, $65,444-455$.

Tobón, J., Vega, M., \& Cuervo, J. (2012). Características de la construcción del vínculo afectivo de pareja en la juventud en la ciudad de Medellín. CES Psicología, 5(1), 49-64.

Tzeng, O., \& Gomez, M. (1992). Octagonal cycle and differential perspectives. Nueva York: Praeger.

Ventura, J., \& Caycho, T. (2016). Análisis exploratorio de la Escala Triangular del Amor en estudiantes universitarios peruanos. Acta de Investigación Psicológica, 6, 2430-2439.

Vera, J., Félix, J., \& Rodríguez, A. (2001). Satisfacción marital, edad, número de hijos y años en pareja. Revista de la Facultad de Psicología de la Universidad Nacional Mayor de San Marcos, 5(1-2), 233-250.

Villamizar, D. (2009). Las representaciones de las relaciones de pareja a lo largo del ciclo vital: significados asociados y percepción del cambio evolutivo (tesis doctoral). Universitat Rovira i Virgili, Tarragona, España.

Villarreal, M. (2016). Intimidad y religiosidad como posibles predictores de satisfacción en la relación conyugal (tesis de maestría). Universidad Pontificia Comillas, Madrid, España.

Witherspoon Institute. (2008). Marriage and the Public Good: Ten Principles. Princeton: Witherspoon Institute. 